\title{
Implementation and performance of the tau trigger in the ATLAS experiment
}

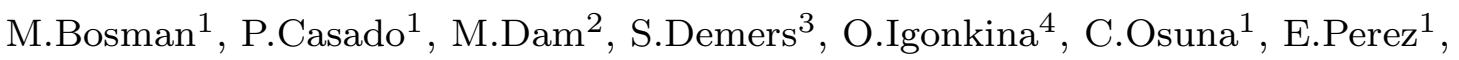 \\ R.Soluk ${ }^{5}$, D.Strom ${ }^{4}$, E.Torrence ${ }^{4}$, A.Watson ${ }^{6}$, S.Xella ${ }^{2} *$ \\ ${ }^{1}$ Institut de Fisica d'Altes Energies (IFAE) \\ Edifici Cn, Campus UAB, E-08193 (Spain) \\ E-mail:bosman@ifae.es \\ ${ }^{2}$ Niels Bohr Institute, University of Copenhagen \\ Blegdamsvej 17, DK-2100, Copenhagen \\ E-mail:xella@nbi.dk \\ ${ }^{3}$ Stanford Linear Accelerator Center (SLAC) \\ 2575 Sand Hill Road, Menlo Park, CA 94025, USA \\ E-mail:demers@slac.stanford.edu \\ ${ }^{4}$ University of Oregon \\ 1226 University of Oregon, Eugene, OR 97403-1226, USA \\ E-mail: strom@physics.uoregon.edu \\ ${ }^{5}$ University of Alberta \\ Edmonton, Alberta, Canada T6G 2G1 \\ E-mail: soluk@phys.ualberta.ca \\ ${ }^{6}$ School of Physics and Astronomy, University of Birmingham \\ Edgbaston, Birmingham B15 2TT, England, UK \\ E-mail: Alan.Watson@cern.ch
}

Triggering on hadronic taus at the LHC is a difficult task due to the high rate and occupancy of the events. On the other hand, the tau trigger increases the discovery potential of ATLAS in many physics channels, among others the Standard Model or SuperSymmetric Higgs (charged or neutrals) production. In order to cope with the rate and optimize the efficiency on important physics channels, the results of the current simulation studies indicate that the ATLAS tau trigger should be used either with relatively high transverse momentum thresholds alone, or with more relaxed threshold requirements in combination with other triggers, like the missing transverse energy trigger or a leptonic or

\footnotetext{
${ }^{*}$ Corresponding author, presenter at ICATPP
} 
jet trigger.

In this contribution we describe the ATLAS tau trigger, and we present some of the current results from the simulation studies, focusing both on early physics and on physics at high luminosity.

Keywords: Atlas; Tau; Trigger.

\section{The ATLAS tau trigger}

In ATLAS, tau leptons are triggered either via the leptonic trigger (electron, muon), when they decay leptonically, or via the tau trigger, when they decay hadronically. In this section we will focus on the latter, describing the working principles and the performance as in the most up to date ATLAS simulation.

\subsection{The ATLAS Level 1 tau trigger}

The Level 1 trigger (LVL1) is a hardware trigger. This implementation satisfies the strong constraints in processing time due to the high input event rate $\sim 40 \mathrm{MHz}$ and the required background rejection factor of 500 . The decision time on an event at this level is of the order of $\mu$ sec.

The way a tau is searched at LVL1 is depicted in Fig. 1 left. A 2x2 tower electromagnetic (EM) cluster and a $2 \times 2$ hadronic (HAD) cluster are used to identify a Region of Interest (RoI). In this core region, a 2x1 or 1x2 tower EM energy plus the $2 \times 2$ HAD energy are required to be above a transverse energy $\left(E_{T}\right)$ threshold. The highest of the 4 possible combinations in the core of the RoI is taken as the energy of the tau candidate. The 12 towers in the outer EM part of the RoI are used to apply isolation criteria.

The hardware scheme for collecting and setting the scale of the tau jet energy at LVL1 inevitably biases and smears the tau transverse energy reconstructed with respect to the true tau visible transverse energy. This results in a rather slow turn-on of the efficiency for taus. This can be seen in Fig. 1 right, showing trigger efficiency for Level 1, and all other levels of the tau trigger, for one of the available tau trigger signatures, tau20i, optimized for taus with visible $E_{T}$ above $20 \mathrm{GeV}$.

The handle for reducing the rate at LVL1 in the tau trigger is high energy threshold requirement for tau candidates. Additionally, missing $E_{T}$ or another lepton or jet trigger can be required. 

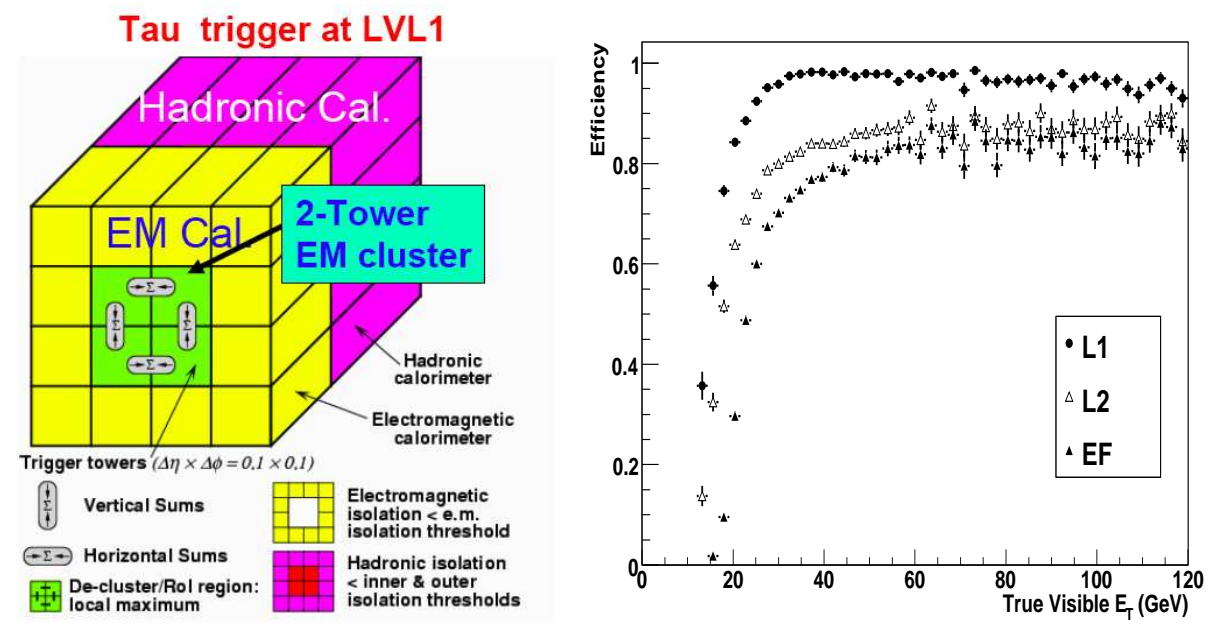

Fig. 1. Region of interest for the Level 1 Tau Trigger (left) and Trigger efficiency on $\mathrm{W} \rightarrow \tau \nu$ events for the trigger signature tau20i (right). Efficiency is normalized to true taus with visible $\mathrm{E}_{T}$ above $20 \mathrm{GeV}$, matched to offline reconstructed tau candidates.

\subsection{High Level Tau Trigger}

The Level 2 Trigger (LVL2) and the Event Filter (EF) parts in the tau trigger are software triggers. More decision time (tens of milliseconds at LVL2, a fraction of a second at EF) and more algorithm flexibility are allowed. The fine granularity and the precision electronics from the calorimeter can be used. Additionally, tracks are reconstructed. Hence signal over background ratio can be enhanced, allowing a richer sample of interesting physics events with $\operatorname{tau}(\mathrm{s})$ in the final state to be saved on tape.

At the second level trigger, the tracking and calorimeter informations collected within a LVL1 RoI are used to created a refined tau candidate. The characteristics of a tau jet, such as low charged track multiplicity, narrowness, isolation, are imposed, to suppress further background QCD jets. Additionally, the energy of the tau candidate is estimated more accurately with respect to LVL1.

At the Event Filter (EF) level, offline reconstruction and calibration procedures for taus are used, on RoIs refined by LVL2. The tau identification used at EF is calorimeter based, analysing the shape of the shower, and matching tracks. An alternative algorithm, which builds a cluster around a reconstructed "leading" track is being developed in analogy to offline reconstruction, in order to better trigger on low $E_{T}$ taus.

According to current studies, an additional background rejection factor of 20 to 50 with respect to LVL1 can be achieved at the HLT, while 
keeping the overall trigger efficiency on signal with respect to good offline reconstructed taus above $60 \%$, for a given $E_{T}$ threshold. This is seen also in Fig. 1 right.

\section{Tau trigger menus for physics}

Some examples of possible tau triggers rates and efficiencies for low $\left(10^{31} \mathrm{~cm}^{-2} \mathrm{~s}^{-1}\right)$ and high $\left(10^{33} \mathrm{~cm}^{-2} \mathrm{~s}^{-1}\right)$ luminosity periods are shown in Tab. 1 and Tab. 2.

The main physics goal of the low luminosity period from the tau trigger point of view is to collect enough data to understand tau identification as soon as possible, and provide a solid backup for discovery searches at higher luminosities using tau leptons in the final state.

Therefore during the low luminosity period the tau trigger aims at collecting a large sample of $\mathrm{W}$ bosons decaying into tau and neutrino, and this can be achieved using a single tau trigger in combination with the request of large missing $E_{T}$ (denoted as MET in tables). This avoids heavy prescaling of the trigger and depletion of the soft $E_{T}$ tau samples from W. Rates will be of the order of $10 \mathrm{~Hz}$ at $10^{31}$.

Additional triggers at low luminosity, providing low rate unprescaled triggers with low $\mathrm{pT}$ requirement, are the egamma/muon combined with tau triggers, requiring two objects in the event. They can be used to collect $\mathrm{Z}$ bosons decaying into two taus, in addition to single electron or muon triggers. The combination of 2 signatures allows to use lower thresholds. A combination of tau trigger and $\mathrm{b}$ jet trigger plus additional jet(s) trigger is under study, and seems to provide a good trigger for taus from top decays in $t \bar{t}$ production.

At higher luminosities single or double tau triggers aim at ensuring high discovery potential for new, heavy particles decaying into tau lepton(s), like SM Higgs or beyond SM charged or neutral Higgs. While the tau combined with missing $E_{T}$ trigger has always been considered the trigger menu for tau final states at high luminosity, recent studies show that double tau triggers or a tau trigger combined with leptonic or jet trigger provide valuable additional triggers. At high luminosity also the more complex signatures like tau plus lepton plus jets or tau plus lepton plus MET will become very important as jets only or MET only might not give a sufficient rate.

A small subset of possible tau trigger menus for different luminosities is shown in Tab. 1 and Tab. 2. The efficiency of the single tau signatures on a tau offline reconstructed candidate matched to a true tau with visible $E_{T}$ and $\eta$ in acceptance is typically $60 \%$. 
Table 1. Example of tau menus for $10^{31} \mathrm{~cm}^{-2} \mathrm{~s}^{-1}$

\begin{tabular}{cccc}
\hline Selection & L1 $(\mathrm{Hz})$ & EF $(\mathrm{Hz})$ & Goal \\
\hline tau20i + MET 30 & 500 & 6 & $\mathrm{~W} \rightarrow \tau \nu$ \\
\hline tau60 & 80 & 11 & high $\mathrm{pt}$ searches \\
\hline tau20i + e10 & 1300 & 1 & $\mathrm{Z} \rightarrow \tau \tau$ \\
\hline tau20i + mu6 & 50 & 3 & $\mathrm{Z} \rightarrow \tau \tau$ \\
\hline tau20i $+3 \mathrm{j} 23$ & 314 & 1 & $t \bar{t}$ \\
\hline
\end{tabular}

Table 2. Example of tau menus for $10^{33} \mathrm{~cm}^{-2} \mathrm{~s}^{-1}$

\begin{tabular}{cccc}
\hline Selection & L1 $(\mathrm{Hz})$ & $\mathrm{EF}(\mathrm{Hz})$ & Goal \\
\hline tau45i + MET 45 & $1.4 \mathrm{~K}$ & 15 & $\mathrm{H}^{ \pm} \rightarrow \tau \nu, \mathrm{H} \rightarrow \tau \tau$, new resonances \\
\hline tau25i + e25i & $10 \mathrm{~K}$ & 10 & $\mathrm{H} \rightarrow \tau \tau$, SUSY particles \\
\hline tau25i + mu20 & $2 \mathrm{~K}$ & 10 & $\mathrm{H} \rightarrow \tau \tau$, SUSY particles \\
\hline
\end{tabular}

\section{Conclusions}

The ATLAS tau trigger is an important trigger for many discovery physics channels at the LHC. A lot of effort within the tau trigger group is devoted to the optimization of the tau trigger selection, and to the trigger menu definition for low and high luminosity periods of data taking, to ensure good unprescaled triggers for important physics channels. 